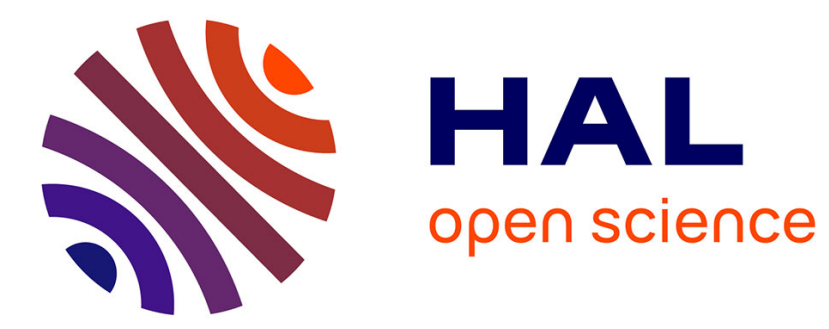

\title{
DYNAMIC LAW OF PHYSICAL MOTION AND POTENTIAL-DESCENDING PRINCIPLE
}

\author{
Tian Ma, Shouhong Wang
}

\section{To cite this version:}

Tian Ma, Shouhong Wang. DYNAMIC LAW OF PHYSICAL MOTION AND POTENTIALDESCENDING PRINCIPLE. 2017. hal-01558752

\section{HAL Id: hal-01558752 \\ https://hal.science/hal-01558752}

Preprint submitted on 9 Jul 2017

HAL is a multi-disciplinary open access archive for the deposit and dissemination of scientific research documents, whether they are published or not. The documents may come from teaching and research institutions in France or abroad, or from public or private research centers.
L'archive ouverte pluridisciplinaire HAL, est destinée au dépôt et à la diffusion de documents scientifiques de niveau recherche, publiés ou non, émanant des établissements d'enseignement et de recherche français ou étrangers, des laboratoires publics ou privés. 


\title{
DYNAMIC LAW OF PHYSICAL MOTION AND POTENTIAL-DESCENDING PRINCIPLE
}

\author{
TIAN MA AND SHOUHONG WANG
}

\begin{abstract}
The main objectives of this paper are five-fold. The first is to introduce a general dynamic law for all physical motion systems, based on a new variational principle with constraintinfinitesimals. The second is to postulate the potential-descending principle (PDP). We show that PDP is a more fundamental principle than the first and second laws in thermodynamics, and gives rise to dynamical equations for non-equilibrium systems. The third is to demonstrate that the PDP is the first principle to describe irreversibility of all thermodynamic systems, with thermodynamic potential as the basic physical quantity, rather than entropy. The fourth objective is to examine the problems faced by the Boltzmann equation. We show that the Boltzmann is not a physical law, is created as a mathematical model to obey the entropy-increasing principle (for dilute gases), and consequently is unable to faithfully describe Nature. The fifth objective is to prove an orthogonal-decomposition theorem and a theorem on variation with constraint-infinitesimals, providing the needed mathematical foundations of the dynamical law of physical motion.
\end{abstract}

\section{Contents}

1. Introduction 2

2. Dynamic Law of Physical Motion 8

$\begin{array}{lll}2.1 . & \text { General guiding principles } & 8\end{array}$

\begin{tabular}{lll}
\hline 2.2. & Principle of symmetry-breaking & 8
\end{tabular}

2.3. Dynamic Law of Physical Motion 9

3. Potential-Descending Principle in Statistical Physics 12

\begin{tabular}{lll}
\hline 3.1. & Thermodynamic potentials & 12
\end{tabular}

Date: July 5, 2017.

Key words and phrases. dynamical law of physical motion, potential-descending principle, statistical physics, thermodynamics, entropy, irreversible processes Boltzmann equation, orthogonal-decomposition theorem, variation with constraintinfinitesimals.

The work was supported in part by the US National Science Foundation (NSF), the Office of Naval Research (ONR) and by the Chinese National Science Foundation. 


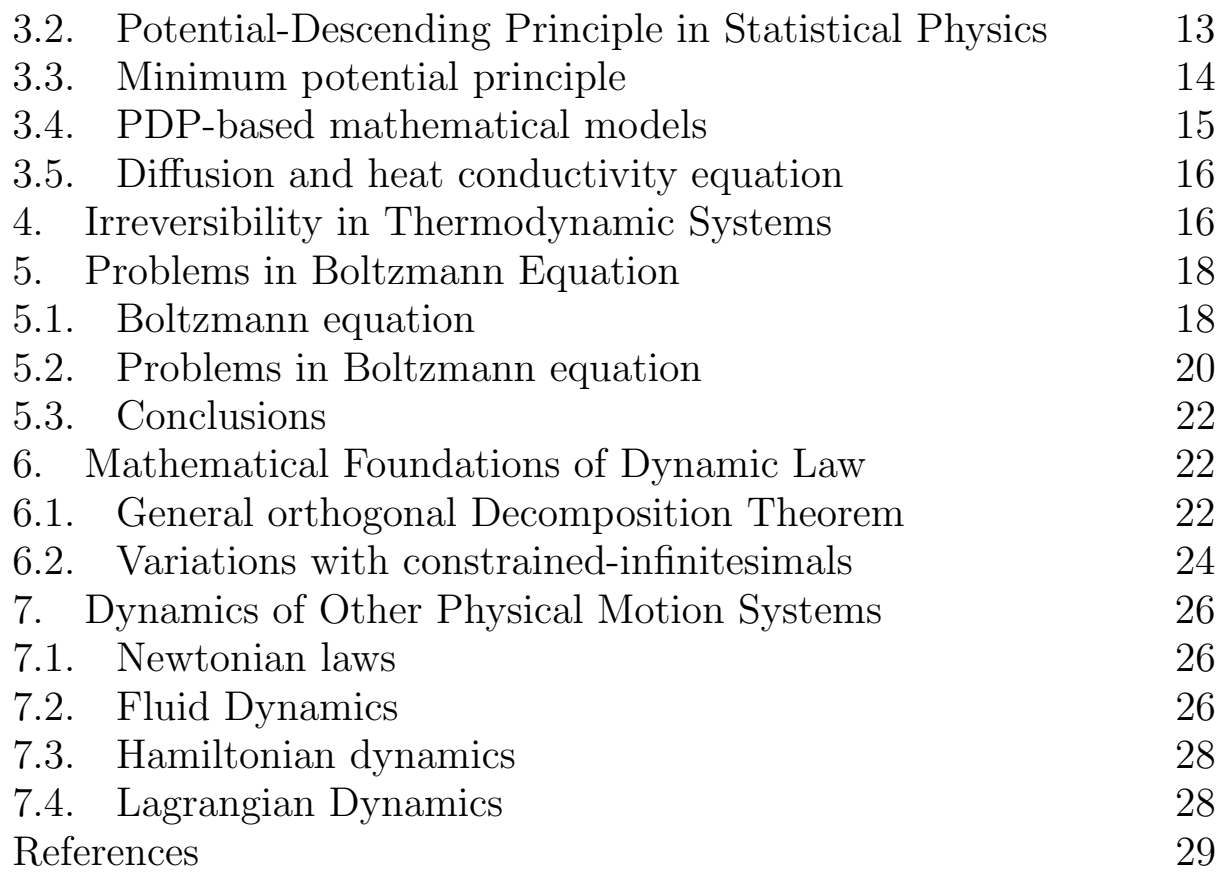

\section{INTRODUCTION}

The heart of physics is to seek experimentally verifiable, fundamental laws and principles of Nature. In this process, physical concepts and theories are transformed into mathematical models and the predictions derived from these models can be verified experimentally and conform to reality. In their mathematical form, the physical laws are often can be expressed as mathematical equations:

$$
\text { physical laws = mathematical equations. }
$$

Among most important physical laws are 1) the laws for fundamental interactions - gravity, electromagnetism, weak and strong - and 2) the laws for motion dynamics. Modern theory of fundamental interactions is based on the field theoretical point of view; see among many others [4] and their references therein.

The focus of this paper is on dynamical laws of physical motion systems. According to their scales, the physical motion systems include 1) classical mechanical systems, describing planetary scale motion, 2) quantum systems for particles in the micro level, 3) fluid mechanics systems, macroscopic description of fluid motion, 4) astrophysical systems for astronomical objects, and 5) statistical systems, relating microscopic properties of individual particles to the macroscopic properties. 
The main objectives of this paper are 1) to introduce a general dynamic law for all physical motion systems, 2) to postulate the potentialdescending principle (PDP), 3) to demonstrate that the potentialdescending principle is the fundamental principle to describe irreversibility of all thermodynamic systems, 4) to indicate the problems faced by the Boltzmann equation, and 5) to prove an orthogonal-decomposition theorem and a theorem on variation with constraint-infinitesimals. Hereafter we now give a brief description the main ingredients of this paper.

\section{Dynamic law of physical motion}

FIRST, for each isolated physical motion system, there are a set of state functions $u=\left(u_{1}, \cdots, u_{N}\right)$, describing the states of the system, and a potential functional $F(u)$, representing a certain form of energy intrinsic to the underlying physical system. Then it is physically clear that the rate of change of the state functions $d u / d t$ should equal to the driving force derived from the potential functional $F$. More precisely, we postulate the following dynamical law of physical motion:

$$
\frac{\mathrm{d} u}{\mathrm{~d} t}=-A \delta_{\mathcal{L}} F(u)
$$

where $A$ is the coefficient matrix, and $-\delta_{\mathcal{L}} F(u)$ is the variation with constraint infinitesimals, representing the driving force of the physical motion system, and $\mathcal{L}$ is a differential operator representing the infinitesimal constraint.

SECOND, we shall demonstrate that proper constraints should be imposed on the infinitesimals (variation elements) for the variation of the energy functional $F$. These constraints can be considered as generalized energy and/or momentum conservation laws for the infinitesimal variation elements.

The variation under constraint infinitesimals is motivated in part by the recent work of the authors on the principle of interaction dynamics (PID) for the four fundamental interactions, which was required by the dark energy and dark matter phenomena, the Higgs fields, and the quark confinement; see [4]. Basically, PID takes the variation of the Lagrangian actions for the four interactions, under energy-momentum conservation constraints.

The precise mathematical description of this constraint variation is one of the main objectives of this paper, and will be addressed in detail near the end of the Introduction.

THIRD, there are two types of physical motion systems: the dissipative systems and the conservation systems. The coefficient matrix $A$ 
is symmetric and positive definite if and only if the system is a dissipative system, and $A$ is anti-symmetry if and only if the system is a conservation system.

FOURTH, symmetry plays a fundamental role in understanding Nature. In [4], we have demonstrated that for the four fundamental interactions, the Lagrangian actions are dictated by the general covariance (principle of general relativity), the gauge symmetry and the Lorentz symmetry; the field equations are then derived using PID as mentioned earlier.

For isolated motion systems, all energy functionals $F$ obey certain symmetries such as $S O(n)(n=2,3)$ symmetry. In searching for laws of Nature, one inevitably encounters a system consisting of a number of subsystems, each of which enjoys its own symmetry principle with its own symmetry group. To derive the basic law of the coupled system, we have demonstrated in [4] the principle of symmetry-breaking (PSB), which is of fundamental importance for deriving physical laws for both fundamental interactions and motion dynamics:

Physical systems in different levels obey different laws, which are dictated by their corresponding symmetries. For a system coupling different levels of physical laws, part of these symmetries must be broken.

In view of this principle, for a system coupling different subsystems, the motion equations become

$$
\frac{\mathrm{d} u}{\mathrm{~d} t}=-A \delta_{\mathcal{L}} F(u)+B(u),
$$

where $B(u)$ represents the symmetry-breaking. As we shall see in Section 4, all physical motions systems coupling different subsystems are governed by the above dynamical law 1.2 .

FIfTH, the dynamical law given by $(1.1)$ and $(1.2)$ is essentially known for motion system in classical mechanics, quantum mechanics and astrophysics; see among others [1, 4]. Thanks to the variation with infinitesimal constraints, the law of fluid motion is now in the form of (1.1) and (1.2). The potential-descending principle given below shows that non-equilibrium thermodynamical systems are governed by the dynamical law (1.1) and (1.2), as well.

\section{Potential-Descending Principle}

After a thorough examination of thermodynamics, we discover that the Potential-Descending Principle (PDP), which we shall postulate below, is a more fundamental principle in statistical principle. 
FIRST, for a given thermodynamic system, the order parameters (state functions) $u=\left(u_{1}, \cdots, u_{N}\right)$, the control parameters $\lambda$, and the thermodynamic potential (or potential in short) $F$ are well-defined quantities, fully describing the system. The potential is a functional of the order parameters, and is used to represent the thermodynamic state of the system. There are four commonly used thermodynamic potentials: the internal energy, the Helmholtz free energy, the Gibbs free energy, and the enthalpy.

We postulate now the potential-descending principle (PDP) as follows; see Principle 3.1 a more complete statement of this principle: For a non-equilibrium state $u\left(t ; u_{0}\right)$ of a thermodynamic system with initial state $u\left(0, u_{0}\right)=u_{0}$,

1) the potential $F\left(u\left(t ; u_{0}\right) ; \lambda\right)$ is strictly decreasing as time evolves;

2) the order parameters $u\left(t ; u_{0}\right)$, as time evolves to infinity, tends to an equilibrium of the system, which is a minimal point of the potential $F$.

SECOND, we show in Section 3.3 that PDP leads to both the first and the second laws of thermodynamics. In the classical thermodynamics theory (e.g. [6]), the perception is that potential-decreasing property can be derived from the first and second laws. However, in the derivations, there is a hidden assumption that at the equilibrium, there is a free-variable in each of the conjugate pairs. For example, in the internal energy system, the entropy $S$ and the generalized displacement $X$ are free variables. This assumption is mathematically equivalent to the potential-descending principle. In other words, the potential-decreasing property cannot be derived if we treat the first and second laws as the only fundamental principles of thermodynamics. We refer Section 3.3 for details. Therefore we reach the following conclusion:

the potential-descending principle leads to both the first and second laws of thermodynamics, and the potentialdescending principle is a more fundamental principle then the first and second laws.

Hereafter we shall also show that PDP provides the first principle for describing irreversibility.

THIRD, PDP provides dynamic equations for a non-equilibrium thermodynamic system, which take exactly the form given by (1.1) or (1.2); see also 3.9 and 3.10 .

\section{Irreversibility}


We know that the motion of all statistical physical system (collection of large number of particles) possesses irreversibility. Classical thermodynamics attributes this irreversibility to entropy-increasing principle. We show in this paper, however, that the Potential-Descending Principle (PDP) is the first principle for irreversibility for all thermodynamic systems. The main reasons are as follows.

FIRST, PDP indeed offers a clear description of the irreversibility of thermodynamical systems. Consider a non-equilibrium initial state $u_{0}$ of the thermodynamic system with order parameters $u$, control parameters $\lambda$ and potential $F$, the PDP amounts to saying that the potential is decreasing:

$$
\frac{d}{d t} F\left(u\left(t ; u_{0}\right) ; \lambda\right)<0 \quad \forall t>0 .
$$

This shows that the state of the system $u\left(t ; u_{0}\right)$ will never return to its initial state $u_{0}$ in the future time. This is exactly the irreversibility.

SECOND, entropy $S$ is a state function, which is the solution of basic thermodynamic equations. Thermodynamic potential is a higher level physical quantity than entropy, and consequently, is the correct physical quantity, rather than the entropy, for describing irreversibility for all thermodynamic systems.

\section{Problems in Boltzmann Equation}

Historically, great effort has been put on establishing a mathematical model that can faithfully describe irreversible processes. The Boltzmann equation is introduced mainly for this purpose with two specific goals: 1) to derive the entropy-ascending principle, and 2) to make the Maxwell-Boltzmann distribution a steady-state solution.

However, the Boltzmann equation faces many problems. After thorough examination of the derivation and properties of the Boltzmann equation, we show that the Boltzmann equation is not a physical law, and consequently is not able to describe faithfully the Nature. Hereafter we list briefly the main problems it faced.

FIRst, laws of physics (equations) should not use state functions, which are themselves governed by physical laws, as independent variables. The Boltzmann equation violates this simple physical rule by using the velocity field as an independent variable.

SECOND, irreversibility is a common characteristic of all dissipative systems, and is not a property of entropy. The Boltzmann equation was aimed to develop a mathematical model using entropy to describe irreversibility. This is an incorrect starting point. 
THIRD, for a gaseous system with potential $F(u, \lambda)$, order parameters $u$ and control parameters $\lambda$, the potential-descending principle gives rise to the following dynamic equation:

$$
\frac{d u}{d t}=-\delta F(u, \lambda)
$$

which offers a complete description of the irreversibility of the gaseous system. Since irreversibility is a macroscopic property, the above physical law (1.3) is the replacement of the Boltzmann equation.

\section{Variation with constraint infinitesimals}

To establish the needed mathematical foundation for the dynamical law of physical motion systems, we need to prove an orthogonal decomposition theorem, Theorem 6.1. Basically, for a linear operator $L: H_{1} \rightarrow H$ between two Hilbert spaces $H$ and $H_{1}$, with dual operator $L^{*}$, any $u \in H$ then can be decomposed as

$$
u=L \varphi+v, \quad L^{*} v=0,
$$

where $L \varphi$ and $v$ are orthogonal in $H$.

The linear operator $\mathcal{L}$ in the dynamic law (1.1) takes the form of a differential operator $L$ or its dual $\mathcal{L}^{*}$. The constraints can be imposed either on the kernel $\mathcal{N}^{*}$ of the dual operator $L^{*}$ or on the range of the operator $L$, given as follows:

$$
\begin{array}{ll}
\left\langle\delta_{L^{*}} F(u), v\right\rangle_{H}=\left.\frac{\mathrm{d}}{\mathrm{d} t}\right|_{t=0} F(u+t v) & \forall L^{*} v=0, \\
\left\langle\delta_{L} F(u), \varphi\right\rangle_{H_{1}}=\left.\frac{\mathrm{d}}{\mathrm{d} t}\right|_{t=0} F(u+t L \varphi) & \forall \varphi \in H_{1} .
\end{array}
$$

Then using the general orthogonal decomposition theorem, Theorem 6.1, we show that the above variations with constraint infinitesimals take the following form:

$$
\begin{aligned}
& \delta_{L^{*}} F(u)=\delta F(u)+L p, \\
& \delta_{L} F(u)=L^{*} \delta F(u),
\end{aligned}
$$

for some function $p$, which plays a similar role as the pressure in incompressible fluid flows. Here $\delta F(u)$ is the usual derivative operator.

The paper is organized as follows. Section 2 introduces the dynamical law of physical motion systems, and Section 3 postulates the potentialdescending principle. Section 4 identifies the physical quantity and principle for irreversibility, and Section 5 points out problems in the Boltzmann equation. Section 6 provides the mathematical foundations 
of the dynamical law of physical motion by proving the orthogonaldecomposition theorem and a theorem on variation with constraintinfinitesimals. Section 7 verifies the dynamical law of physical motions by examining various important motion dynamical equations.

\section{Dynamic Law of Physical Motion}

2.1. General guiding principles. For theoretical physics, the following general guiding principle was introduced in [4]:

All physical systems obey laws and principles of Nature, and possess the following three properties:

1) For each system, there is a group of functions $u=$ $\left(u_{1}, \cdots, u_{N}\right)$ describing its states, and the laws and principles obeyed by the system can be expressed as: physical laws = mathematical equations, with the state functions $u$ as solutions;

2) For each system, there is a functional $F(u)$, which dictates mathematical equations;

3) All physical systems obey certain symmetries, which essentially determine the mathematical forms of the functionals $F$.

2.2. Principle of symmetry-breaking. Symmetry plays a fundamental role in understanding Nature. In mathematical terms, each symmetry, associated with particular physical laws, consists of three main ingredients: 1) the underlying space, 2) the symmetry group, and 3) tensors, describing the objects which possess the symmetry [4].

One important point to make is that different physical systems enjoy different symmetries. For example, gravitational interaction enjoys the symmetry of general relativity, which, amazingly, dictates the Lagrangian action for the law of gravity. Also, the other three fundamental interactions obey the gauge and the Lorentz symmetries, which dictate also the corresponding actions. The Newtonian motion systems obey the Galileo symmetry, and all motion systems satisfy the $S O(n)$ symmetry.

In searching for laws of Nature, one inevitably encounters a system consisting of a number of subsystems, each of which enjoys its own symmetry principle with its own symmetry group. To derive the basic law of the system, we have demonstrated in [4] the following principle of symmetry-breaking (PSB), which is of fundamental importance for deriving physical laws for both fundamental interactions and motion dynamics. We recapitulate this principle as follows. 
Principle 2.1 (PSB). Physical systems in different levels obey different laws, which are dictated by corresponding symmetries. For a system coupling different levels of physical laws, part of these symmetries must be broken:

1) There are several main symmetries- - the $S O(n)$ invariance, the Galileo invariance, the Lorentz invariance, the $S U(N)$ gauge invariance, and the general relativistic invariance- which are mutually independent and dictate in part the physical laws in different levels of Nature; and

2) for a system coupling several different physical laws, part of these symmetries must be broken.

2.3. Dynamic Law of Physical Motion. Theoretical physics studies 1) the laws for the four fundamental interactions (gravity, electromagnetism, the weak and the strong interactions), and 2) the laws for motion dynamics of physical systems.

According to their scales, physical systems are classified into

(1) classical mechanical systems (planetary scale),

(2) quantum systems (micro level),

(3) fluid mechanics systems,

(4) astrophysical systems, and

(5) statistical systems (relating microscopic properties of individual particles to the macroscopic properties).

The basic laws for describing classical mechanical systems, quantum systems and astrophysical systems are given in terms of Newtonian laws, the principle of Lagrangian dynamics and the principle of Hamiltonian dynamics. We shall see in Section 3 below that statistical physical systems are described by the potential-descending principle. In Section 7, we show that fluid motion satisfies the dynamical law of physical motion introduced in the following.

By a careful examination of basic motion laws of different physical systems and by the mathematical study in Section 6 below, we discover a general dynamic law, suitable for all physical motion systems in (1)(5) mentioned above.

To state this general dynamic law, we start with the definition of constraint variation. Let $H, H_{1}$ be two Hilbert spaces, and

$$
L: H_{1} \rightarrow H, \quad L^{*}: H \rightarrow H_{1}^{*}
$$

be a pair of dual linear bounded operators. Consider a functional defined on $H$ :

$$
F: H \rightarrow \mathbb{R}^{1} .
$$


Let

$$
\mathcal{N}^{*}=\left\{v \in H \mid L^{*} v=0\right\} \neq\{0\} .
$$

Definition 2.1. Let (2.3) hold.

1) For any $u \in H$, the derivative operator of $F$ at $u$ with $L^{*}$-constraint, denoted by $\delta_{L^{*}} F(u)$, is defined as follows

$$
\left\langle\delta_{L^{*}} F(u), v\right\rangle_{H}=\left.\frac{d}{d t}\right|_{t=0} F(u+t v) \quad \forall v \in \mathcal{N}^{*} .
$$

2) The derivative operator of $F$ at $u$ with $L$-constraint, denoted by $\delta_{L} F(u)$, is defined as follows

$$
\left\langle\delta_{L} F(u), \varphi\right\rangle_{H_{1}}=\left.\frac{d}{d t}\right|_{t=0} F(u+t L \varphi) \quad \forall \varphi \in H_{1} .
$$

Further properties of constrained variations and the general orthogonal decomposition theorem will be introduced in Section 6. We are now ready to state the dynamical law of physical motion.

Dynamical Law 2.2. There are two types of physical motion systems: dissipative systems and conservation systems. For each physical motion system, there are a set of state functions $u=\left(u_{1}, \cdots, u_{N}\right)$, a functional $F(u)$, and an operator $\mathcal{L}$, which is either $\mathcal{L}=L$ or $\mathcal{L}=L^{*}$ for some differential operator $L$, such that the following statements hold true:

1) $-\delta_{\mathcal{L}} F(u)$ is the deriving force of the system;

2) $F$ is $S O(n)(n=2,3)$ invariant;

3) for an isolated system, the dynamic equation can be expressed in the form

$$
\frac{d u}{d t}=-A \delta_{\mathcal{L}} F(u)
$$

where $A$ is the coefficient matrix, $A$ is symmetric and positive definite if and only if the system is a dissipative system, and $A$ is anti-symmetry if and only if the system is a conservation system;

4) for a system coupling different subsystems, the motion equations of (2.6) become

$$
\frac{d u}{d t}=-A \delta_{\mathcal{L}} F(u)+B(u)
$$

where $B(u)$ represents the symmetry-breaking.

A few remarks are in-order. 
First, in (2.6) and (2.7), the derivative $\mathrm{d} / \mathrm{d} t$ is physical, namely

$$
\begin{array}{ll}
\frac{\mathrm{d}}{\mathrm{d} t}=\frac{\partial}{\partial t}+i g \mathbf{A}_{0} & \text { for gauge fields }, \\
\frac{\mathrm{d}}{\mathrm{d} t}=\frac{\partial}{\partial t}+(u \cdot \nabla) & \text { for fluid fields. }
\end{array}
$$

Second, the operator $\mathcal{L}\left(=L\right.$ or $\left.L^{*}\right)$ in physical systems is an operator, and when $\mathcal{L}=i d$ is the identity operator, $\delta_{\mathcal{L}}$ is the usual derivative operator. Typical form of the operator $L$ is the gradient operator with $L^{*}$ being the corresponding divergence-operator.

Third, $-\delta_{\mathcal{L}} F(u)$ represents the generalized force, and $(2.6)$ is the generalized Newtonian Law. For example, consider the classical Newtonian Second Law:

$$
\frac{d^{2} x}{d t^{2}}=-\nabla \varphi
$$

where $\varphi$ is an interaction potential. The equation $(2.8)$ is equivalent to

$$
\frac{d x}{d t}=y, \quad \frac{d y}{d t}=-\nabla \varphi
$$

Now let

$$
\Phi=\frac{y^{2}}{2}+\varphi, \quad u=(x, y)^{T}
$$

Let

$$
J=\left(\begin{array}{cc}
0 & -I \\
I & 0
\end{array}\right)
$$

be the symplectic matrix. Then we have

$$
J \nabla \Phi=(-y, \nabla \varphi)^{T},
$$

and the Newtonian Second Law can be rewritten in the form of 2.6 as follows:

$$
\frac{d u}{d t}=-J \nabla \Phi
$$

Here there is no constraint; namely, the operator $\mathcal{L}=L$ is the identity operator.

Fourth, the $B(u)$ term in (2.7) represents the symmetry-breaking. For example, consider the damped wave equation

$$
u_{t t}=\Delta u-k u_{t}+f(u) .
$$

Let $v=u_{t}$ and $\psi=(u, v)^{T}$, then the wave equation can be written as

$$
\frac{d}{d t} \psi=-J \delta F(\psi)+B \psi
$$




$$
\begin{aligned}
& F(\psi)=\int\left[\frac{1}{2} v^{2}+|\nabla u|^{2}+G(u)\right], \\
& B \psi=\left(\begin{array}{c}
0 \\
-k v
\end{array}\right) .
\end{aligned}
$$

Here the functional $F$ inherits the symmetry of the wave equation without damping term $k u_{t}$. But the damping term cannot be included in the functional $F$, and breaks the symmetry of the non-damped wave equation.

As mentioned earlier, symmetry-breaking is the essence for modeling a physical system coupling subsystems obeying different symmetries.

\section{Potential-Descending Principle in Statistical Physics}

In this section, we postulate the potential-descending principle (PDP) in statistical physics, which is more fundamental than the first and the second laws of thermodynamics. This principle also supports the dynamic law of physical motion introduced in the last section.

3.1. Thermodynamic potentials. A thermodynamic system is described by order parameters (state functions), control parameters, and thermodynamic potential, which is a functional of the order parameters. There are four commonly used thermodynamic potentials: the internal energy, the Helmholtz free energy, the Gibbs free energy, and the enthalpy. The basic thermodynamic potential is the internal energy, and the other three are derived using the Legendre transforms; see among others [6].

Conjugate pairs of state variables and internal energy. First, we note that all thermodynamic potentials are expressed in terms of conjugate pairs. The most commonly considered conjugate thermodynamic variables are

1) the temperature $\mathrm{T}$ and the entropy $\mathrm{S}$, and

2) $f$ the generalized force and $X$ the generalized displacement. Typical examples of $(f, X)$ include (the pressure $p$, the volume $V$ ), (applied magnetic field $H$, magnetization $M$ ), (applied electric field $E$, electric polarization $P$ ).

Let $U$ be the internal energy. Then for a completely closed thermodynamic system, the first law states:

$$
d U=T d S+f d X .
$$

Here $(T, f)$ are the order parameters and $(S, X)$ are the control parameters. 
Helmholtz energy. For an isothermal process, we need to use the Helmholtz free energy, denoted by

$$
F=U-T S \text {. }
$$

In fact, 3.2 is the Legendre transformation such that the Helmholtz free energy $F$ is a functional of $S$ and $f$. In other words, $(S, f)$ are order parameters, and $(T, X)$ are control parameters. Consequently,

$$
F=F(S, f ; \lambda), \quad \lambda=(T, X),
$$

and the equilibrium state enjoys

$$
\frac{\delta F}{\delta S}=0, \quad \frac{\delta F}{\delta f}=0 .
$$

Gibbs free energy. When the system has the thermal and mechanical exchanges with the external, i.e., the thermal process is in the constant temperature $T$ and generalized force $f$, the corresponding potential is the Gibbs free energy, which is defined by

$$
G=F-f X, \quad F \text { the Helmholtz free energy, }
$$

which is the Legendre transformation regarding $(T, f)$ as its independent variables. Namely, for the Gibbs potential, the order parameters are $(S, X)$, and the control parameters are $\lambda=(T, f)$,

$$
G=G(S, X ; \lambda)
$$

and the equilibrium state satisfies

$$
\frac{\delta G}{\delta S}=0, \quad \frac{\delta G}{\delta X}=0 .
$$

3.2. Potential-Descending Principle in Statistical Physics. We postulate the following potential descending principle (PDP), and demonstrate in the remaining part of this paper that this principle serves as a fundamental principle in statistical physics.

Principle 3.1 (Potential-Descending Principle). For each thermodynamic system, there are order parameters $u=\left(u_{1}, \cdots, u_{N}\right)$, control parameters $\lambda$, and the thermodynamic potential functional $F(u ; \lambda)$. For a non-equilibrium state $u\left(t ; u_{0}\right)$ of the system with initial state $u\left(0, u_{0}\right)=$ $u_{0}$, we have the following properties:

$1)$ the potential $F\left(u\left(t ; u_{0}\right) ; \lambda\right)$ is decreasing:

$$
\frac{d}{d t} F\left(u\left(t ; u_{0}\right) ; \lambda\right)<0 \quad \forall t>0 ;
$$

2) the order parameters $u\left(t ; u_{0}\right)$ have a limit

$$
\lim _{t \rightarrow \infty} u\left(t ; u_{0}\right)=\bar{u}
$$


3) there is an open and dense set $\mathcal{O}$ of initial data in the space of state functions, such that for any $u_{0} \in \mathcal{O}$, the corresponding $\bar{u}$ is a minimum of $F$, which is called an equilibrium of the thermodynamic system:

$$
\delta F(\bar{u} ; \lambda)=0
$$

where $\delta F$ represents the generalized force given in (2.6) and 2.7. Namely,

$$
\delta F(\bar{u} ; \lambda)=-A \delta_{\mathcal{L}} F(\bar{u} ; \lambda) .
$$

3.3. Minimum potential principle. For an equilibrium state $\bar{u}$ of a thermodynamic system, the potential-descending principle implies that

$$
\begin{aligned}
& \frac{\delta}{\delta u} F(\bar{u} ; \lambda)=0, \\
& d F(\bar{u}, \lambda)=\frac{\partial F(\bar{u}, \lambda)}{\partial \lambda} d \lambda .
\end{aligned}
$$

These are equilibrium equations of the system.

For the equilibrium state, it is then easy to see that

$$
d F(\bar{u}, \lambda)=\frac{\delta}{\delta u} F(\bar{u} ; \lambda) \delta u+\frac{\partial F}{\partial \lambda} d \lambda=\frac{\partial F(\bar{u} ; \lambda)}{\partial \lambda} d \lambda,
$$

which is the first law of thermodynamics.

For a given non-equilibrium thermodynamic state $u(t)$, we have

$$
d F(u(t), \lambda)=\frac{\delta}{\delta u} F(u(t) ; \lambda) d u+\frac{\partial F}{\partial \lambda} d \lambda .
$$

The potential-descending principle tells us that

$$
\frac{d F}{d t}=\frac{\delta}{\delta u} F(u(t) ; \lambda) \frac{d u}{d t}<0 .
$$

As $d t>0$, we then derive that

$$
d F(u, \lambda)<\frac{\partial F}{\partial \lambda} d \lambda
$$

which is the second law of thermodynamics.

Also, as an example, we consider an internal energy of a thermodynamic system, classical theory asserts that the first and second laws are given by

$$
d U \leq \frac{\partial U}{\partial S} d S+\frac{\partial U}{\partial X} d X
$$

where the equality represents the first laws, describing the equilibrium state, and inequality presents second law for non-equilibrium state. However, there is a hidden assumption in 3.8 that at the equilibrium, 
there is a free-variable in each of the conjugate pairs $(T, S)$ and $(f, X)$. In the internal energy system, $S$ and $X$ are free variables. Namely,

$$
\frac{\partial U}{\partial T} \leq 0, \quad \frac{\partial U}{\partial f} \leq 0,
$$

where, again, the equality is for equilibrium state and the strict inequality is for non-equilibrium state. This assumption is mathematically equivalent to the minimal-potential principle (or potential-descending principle).

In a nutshell, based on the above discussions, we have the following important conclusion:

the potential-descending principle leads to both the first and second law of thermodynamics, and the potentialdescending principle is a more fundamental principle then the first and second laws.

3.4. PDP-based mathematical models. Based on the principle, the dynamic equations of a thermodynamic system in non-equilibrium state take the form

$$
\begin{aligned}
& \frac{\mathrm{d} u}{\mathrm{~d} t}=-A \delta_{\mathcal{L}} F(u) \quad \text { for isolated systems, } \\
& \left\{\begin{array}{l}
\frac{\mathrm{d} u}{\mathrm{~d} t}=-A \delta_{\mathcal{L}} F(u)+B(u), \\
\int A \delta_{\mathcal{L}} F(u) \cdot B(u)=0
\end{array} \quad\right. \text { for coupled systems, }
\end{aligned}
$$

where $\delta_{\mathcal{L}}$ is the derivative operator with $\mathcal{L}$ - constraint for some operator $\mathcal{L}, B$ represents coupling operators, and $A$ is a symmetric and positive definite matrix of coefficients.

Consider a thermodynamic system involving a conserved order parameter $u$ :

$$
\int_{\Omega} u(x, t) d x=\text { constant. }
$$

The potential is given by

$$
F=F_{1}-\mu \int_{\Omega} u d x
$$

where $\mu$ is the chemical potential, which plays the role of Lagrangian multiplier, and $F_{1}$ is the classical thermodynamical potential of the system. The key point here is that chemical potential cannot be regarded either as an order parameter or as a control parameter for the thermodynamic system. Consequently, the model based on the potentialdescending principle is still given by $(3.9)$ and $(3.10)$. 
It is important to remark that in [3] we used

$$
\begin{aligned}
& \frac{\partial u_{j}}{\partial t}=-\nabla \cdot J_{j}(u, \lambda), \\
& J=-k \nabla \mu \\
& \mu=\frac{\delta F}{\delta u}
\end{aligned}
$$

to derive the following dynamical equation; see [3, (3.1.21)]:

$$
\frac{\partial u}{\partial t}=\Delta \frac{\delta}{\delta u} F(u, \lambda)
$$

However, we realized in this article that the dynamical equation (3.16) (the model (3.1.21) in [3]), as well as the classical Cahn-Hilliard equation, are incorrect. In fact, based on the Lagrangian multiplier theorem and by (3.11) and (3.15), we deduce that $\mu$ is the Lagrangian multiplier, which is a constant. Therefore, the relation (3.14) is invalid.

3.5. Diffusion and heat conductivity equation. Diffusion and heat conduction are properties of thermal systems. Their potential functional is written as

$$
F=\int_{\Omega}\left[\frac{\kappa}{2}|\nabla T|^{2}-Q T\right] \mathrm{d} x
$$

where $u$ represents the density for diffusion systems and the temperature for heat conduction systems. Consequently, the dynamic equation

$$
\frac{\mathrm{d} T}{\mathrm{~d} t}=-\delta F(T)
$$

is in the form:

$$
\frac{\partial T}{\partial t}=\kappa \Delta T+Q
$$

\section{Irreversibility in Thermodynamic Systems}

Natural phenomena show that the motion of all statistical physical system (collection of large number of particles) possesses irreversibility. Namely, when the system evolves from one initial state to another, the system will not spontaneously return to its initial state without external interference. This phenomena is called (temporal) irreversibility.

Classical thermodynamics attributes this irreversibility to entropyascending principle. Based on the discussions in the previous sections, 
the physical quantity depicting irreversibility is thermodynamical potential. The Potential-Descending Principle (PDP), Principle 3.1, offers a clear description of the irreversibility of thermodynamical systems.

More precisely, consider a thermodynamic system with potential $F(u, \lambda)$, order parameters $u$, control parameters $\lambda$ and a non-equilibrium initial state $u_{0}$. Then the PDP amounts to saying that the potential is decreasing:

$$
\frac{d}{d t} F\left(u\left(t ; u_{0}\right) ; \lambda\right)<0 \quad \forall t>0 .
$$

This shows that the state of the system $u\left(t ; u_{0}\right)$ will never return to its initial state $u_{0}$ in the future time. This is exactly the (temporal) irreversibility.

From the PDP-based mathematical model:

$$
\begin{aligned}
& \frac{d u}{d t}=-\delta F(u ; \lambda), \\
& \left.u\right|_{t=0}=u_{0},
\end{aligned}
$$

we also have a clear understanding of irreversibility. For example, given a non-homogeneous system, its thermodynamic potential is

$$
F=\int_{\Omega}\left[\frac{\kappa}{2}|\nabla u|^{2}+g(u)\right] \mathrm{d} x
$$

and the corresponding equation 4.1 is given by

$$
\begin{aligned}
& \frac{d u}{d t}=\kappa \Delta u-f(u), \\
& \left.u\right|_{t=0}=u_{0},
\end{aligned}
$$

where $f(z)=g^{\prime}(z)$. If we take time reversal transformation $t \rightarrow-t$, then 4.2 can be written as

$$
\begin{aligned}
& \frac{d u}{d t}=-\kappa \Delta u-f(u), \\
& \left.u\right|_{t=0}=u_{0} .
\end{aligned}
$$

Basic theory of partial differential equations shows that for general initial data $u_{0}$, 4.3 has no solution for $t>0$. This is another mathematical description for irreversibility. This description is also suitable for all dissipative systems, including fluid motion. In other words, irreversibility is a fundamental feature of dissipative systems.

It is worth emphasizing here that for isolated simple gas system, the thermodynamic potential is

$$
F=U_{0}-S T-\mu_{1} N-\mu_{2} E,
$$


where $U_{0}$ is the internal energy, which is a constant, $N$ is the number of particles, $\mu_{1}$ and $\mu_{2}$ are Lagrangian multipliers, and $E$ is the total energy. For this system, the entropy $S$ is an order parameter, and temperature $T$ is a control parameter.

By (4.4), we see that potential-descending is equivalent to entropy ascending:

$$
\frac{d}{d t} F(S(t))<0 \quad \Longleftrightarrow \quad \frac{d}{d t} S(t)>0
$$

In addition, according to the entropy formula:

$$
S=k \ln W
$$

and by the minimum potential principle:

$$
\delta F=0,
$$

we can derive, with similar procedures as in [5, Section 6.1], all three distributions: the Maxwell-Boltzmann distribution, the Fermi-Dirac distribution and the Bose-Einstein distribution. This shows that

the potential-descending principle is also the first principle of statistical mechanics.

\section{Problems in Boltzmann Equation}

5.1. Boltzmann equation. In statistical physics, a classical problem is how to establish a mathematical model that can faithfully describe irreversible processes. The Boltzmann equation is introduced mainly for this purpose. We start with a brief introduction on the derivation of the Boltzmann equation.

Consider a system of ideal gases that is in a non-equilibrium state, and assume its dynamical equation takes the form:

$$
\frac{d \rho}{d t}=G(\rho)
$$

where $\rho$ stands for the probability density function of the gas molecules.

By the general guiding principle of physics, (5.1) should be established based on some fundamental laws and/or principles. Classical theories lack of such principles and laws. Therefore the Boltzmann equation is based on fulfilling the following two goals:

(1) Entropy-ascending principle: equation (5.1) should yield entropyascending property:

$$
\frac{d}{d t} S(\rho(t))>0,
$$


where $S(\rho)$ is the entropy functional in terms of $\rho$. Physically, we know that

$$
S=-\int \rho \ln \rho d x d v+S_{0},
$$

where $S_{0}$ is a constant, and $v$ is the velocity of molecules.

(2) Since the Maxwell distribution

$$
\rho_{0}=A e^{-v^{2} / 2 m k T}
$$

is the equilibrium state of ideal gases. This requires that $\rho_{0}$ is a steady state solution of (5.1):

$$
G\left(\rho_{0}\right)=0 .
$$

The requirement (2) above shows that the probability density function $\rho$ must be a function using both the space time coordinate $(t, x)$ and velocity $v$ as independent variables:

$$
\rho=\rho(x, t, v) .
$$

Consequently the total derivative on the left-hand side of $(5.1)$ becomes

$$
\frac{d \rho}{d t}=\frac{\partial \rho}{\partial t}+\frac{\partial \rho}{\partial x} \frac{d x}{d t}+\frac{\partial \rho}{\partial v} \frac{d v}{d t}
$$

Since

$$
\frac{d x}{d t}=v, \quad \frac{d v}{d t}=\frac{F}{m} \quad(\text { Newtonian Second Law })
$$

we derive that

$$
\frac{d \rho}{d t}=\frac{\partial \rho}{\partial t}+v \cdot \nabla \rho+\frac{F}{m} \cdot \frac{\partial \rho}{\partial v} .
$$

Next in order to obtain the expression $G$ in the right-hand-side of (5.1), one needs to use phenomenological approach to come up with a formulation for $G$ satisfying the above two requirements. Boltzmann obtained the following formula for $G$; see [2]:

$$
G(\rho)=\int\left[\rho^{\prime} \rho_{1}^{\prime}-\rho \rho_{1}\right] \omega^{\prime} d \Gamma_{1} d \Gamma^{\prime} d \Gamma_{1}^{\prime},
$$

which is used to explain the change due to collisions.

Then (5.1) can be written as

$$
\frac{\partial \rho}{\partial t}+v \cdot \nabla \rho+\frac{F}{m} \cdot \frac{\partial \rho}{\partial v}=\int\left[\rho^{\prime} \rho_{1}^{\prime}-\rho \rho_{1}\right] \omega^{\prime} d \Gamma_{1} d \Gamma^{\prime} d \Gamma_{1}^{\prime},
$$

which is the famous Boltzmann equation. 
5.2. Problems in Boltzmann equation. As indicated by [6, S11.A. Beyond the Boltzmann equation], computer experiments and a study of correlation functions have shown that the picture of transport phenomena given by Boltzmann is not completely correct. Based on PDP presented earlier in this paper, we are now able to demonstrate more precisely the mathematical and physical problems faced by the Boltzmann equation.

1. Boltzmann equation is not a physical law. The Boltzmann equation is not established on fundamental principles and laws. The physical meaning of $(5.8)$ is simply

$$
\text { the rate of change of } \rho=\text { rate of change due to collisions. }
$$

The equality here is the outcome of pure imagination. In fact,

$$
\text { collision }=\text { very close distance interaction between particles. }
$$

On the other hand, by Principle 2.2, the real physical law should be

$$
\begin{aligned}
& \text { the rate of change of } \rho \\
& =\text { the driving force induced by } \\
& \quad \text { the total interaction potential between particles. }
\end{aligned}
$$

Comparing (5.9)-(5.10) and (5.11), we see that there is a large deviation between the Boltzmann equation and the real physical law.

2. The Boltzmann equation uses the velocity field $v$ as an independent variable, but $v$ itself is a state function. This inevitably induces large deviation between the Boltzmann equation and the reality. There is a force field in the Boltzmann equation (5.8):

$$
F=F(t, x, v),
$$

where $v$ is velocity field of particles. Here $F$ should be a function of $v$, since the velocity field $v$ is treated as an independent variable. This force field is the sum of the external force and the force generated by the total interaction potential of all particles in the system, which includes the force due to collision. Hence $F$ is a new unknown function, and

$$
\text { the Boltzmann equation } 5.8 \text { is incomplete, }
$$
with another unknown function $F$.

3. In order to ensure that the Maxwell distribution $\rho_{0}$ given by (5.4) is a steady state solution of the Boltzmann equation (5.8), one has to assume that $d \rho_{0} / d t=0$, which implies that

$$
\frac{F}{m} \cdot \frac{\partial \rho_{0}}{\partial v}=0 \quad \Longleftrightarrow \quad F \cdot v=0 .
$$


This requires that

$$
F=0 \text {. }
$$

This assumption (5.15) is non-physical, since under (5.15), all particles in the system, with zero force, will make uniform rectilinear motion. This is in contradiction with the reality, and leads to the following inconsistency of the Boltzmann equation with the real phenomena:

the Maxwell distribution (5.4) is a steady state solution of the Boltzmann equation (5.8) under

the non-physical assumption (5.15).

4. In deriving the H-Theorem (i.e. the entropy-ascending principle), the following must be assumed:

$$
F=F(t, x) \text { is independent of } v .
$$

However, the Boltzmann equation (5.8) treats the velocity $v$ as an independent variable. In this case, the force field $F$ must be treated as a function of $v$ as well. Consequently the above assumption (5.17) is rather arbitrary, and any model using velocity $v$ as an independent variable is inconsistent with the assumption (5.17). In other words, the $\mathrm{H}$-Theorem is not a natural consequence of the Boltzmann equation (5.8).

Remark 5.1. In statistical physics, one often regards the Boltzmann equation as a model only suitable for dilute ideal gas. The misperception is that the mutual interaction between particles in a dilute gas system is negligible, leading to the assumption $F=0$ in (5.15). However, the force field generated by the total interaction potential $\Phi$ of all particles:

$$
F=-\nabla \Phi
$$

is not small, and is not simply measured by $r^{-2}$ between particles. The motion of particles in any dilute gas system is chaotic, and the velocities of the particles are not small even when there is no external force present. Consequently, the interaction force field is not small, and cannot simply be ignored.

5. Ignoring the non-physical nature of assumption (5.15), the space of all steady state solutions of the Boltzmann equation (5.8) have 5dimension. Namely, the general form of the steady state solutions is

$$
\bar{\rho}=e^{\alpha_{0}+\alpha_{1} v_{1}+\alpha_{2} v_{2}+\alpha_{3} v_{3}+\alpha_{4} v^{2}},
$$


where $\alpha_{i}(i=0, \cdots, 4)$ are constants, and $v=\left(v_{1}, v_{2}, v_{3}\right)$. This shows that each steady-state is not stable, and this does not fit the reality.

6. Entropy-increasing principle shows that a gaseous system in the equilibrium has the maximum entropy, i.e. the Maxwell distribution (5.4) should be the maximum of (5.3). However, the maximum of (5.3) is given by

$$
\rho_{0}=e^{-1}
$$

Again, this is non-physical.

5.3. Conclusions. In a nutshell, the above discussions clearly demonstrate the following conclusions:

1. Laws of physics (equations) should not use state functions as independent variables, which are themselves governed by physical laws. The Boltzmann equation violates this simple physical rule, and therefore is not able to faithfully reflect the true nature of the underlying physical phenomena.

2. Irreversibility is a common characteristic of all dissipative systems, and is not an entropy property. The Potential-Descending Principle introduced in this paper is the basic mathematical model/description of irreversibility.

3. The Boltzmann equation was aimed to develop a mathematical model for entropy-increasing principle. This is an incorrect starting point, since thermodynamic potential is the right physical quantity for the mathematical characterization of irreversibility, rather than entropy.

4. Let $F(u, \lambda)$ be the thermodynamic potential of a gaseous system with order parameters $u$ and control parameters $\lambda$. Then PDP gives rise to the following dynamic equation:

$$
\frac{d u}{d t}=-\delta F(u, \lambda)
$$

This equation offers a complete description of the irreversibility of the gaseous system.

\section{Mathematical Foundations of Dynamic LaW}

6.1. General orthogonal Decomposition Theorem. The following is a general orthogonal decomposition theorem. Let $X$ be a linear space, $H$ be a Hilbert space, and

$$
L: X \rightarrow H \text { be a linear map. }
$$


Let

$$
\mathcal{N}=\{\varphi \in X \mid L \varphi=0\},
$$

be the kernel of $L$, and let $H_{1}$ be the completion of $X / \mathcal{N}$ with the following norm

$$
\|\varphi\|_{H_{1}}^{2}=\langle L \varphi, L \varphi\rangle_{H}, \quad \varphi \in X / \mathcal{N} .
$$

It is clear that $H_{1}$ is a Hilbert space, and

$$
\begin{aligned}
& L: H_{1} \rightarrow H \text { is bounded, } \\
& L^{*}: H \rightarrow H_{1}^{*} \text { is the dual operator of } L .
\end{aligned}
$$

Then we have the $L$-orthogonal decomposition theorem as follows.

Theorem 6.1 (L-Orthogonal Decomposition Theorem). For the linear operators $L$ and $L^{*}$ in (6.2) and any $u \in H$, there exists $a \varphi \in H_{1}$ and $v \in H$, such that $u$ can be decomposed as

$$
u=L \varphi+v, \quad L^{*} v=0, \quad\langle L \varphi, v\rangle_{H}=0 .
$$

Proof. For a given $u \in H$, consider the existence of the equation

$$
L^{*} L \varphi=L^{*} u
$$

for solution $\varphi \in H_{1}$. By 6.1 we can see that the operator

$$
A=L^{*} L: H_{1} \rightarrow H_{1}^{*}
$$

is positive definition, i.e., for any $\psi \in H_{1}$ we have

$$
\langle A \psi, \psi\rangle_{H_{1}}=\langle L \psi, L \psi\rangle_{H}=\|\psi\|_{H_{1}}^{2} .
$$

Therefore, by the Lax-Milgram theorem, the equation (6.4) has a unique solution $\varphi \in H_{1}$. Then we take

$$
v=u-L \varphi \in H .
$$

By (6.4) we see that

$$
L^{*} v=0
$$

Thus, 6.5 is the decomposition of (6.4), and by 6.6 we have

$$
\langle L \varphi, v\rangle_{H}=\left\langle\varphi, L^{*} v\right\rangle_{H_{1}}=0,
$$

i.e., $L \varphi$ and $v$ are orthogonal in $H$. The proof is complete.

We demonstrate the applications of the above theorem with two examples. 
Example 6.1. Let $H=L^{2}\left(\Omega, \mathbb{R}^{n}\right)$ be the space of all n-dimensional vector fields defined on an open set $\Omega \subset \mathbb{R}^{n}$, and

$$
H_{1}=H^{1}(\Omega) / \mathbb{R}=\left\{\varphi \in H^{1}(\Omega) \mid \int_{\Omega} \varphi \mathrm{d} x=0\right\} .
$$

Let

$$
L=\nabla: H_{1} \rightarrow H \text { be the gradient operator, }
$$

and the duality $L^{*}: H \rightarrow H_{1}^{*}$ is as follows

$$
\left\langle L^{*} v, \varphi\right\rangle_{H_{1}}=\int_{\Omega}(-\operatorname{div} v) \varphi \mathrm{d} x+\int_{\partial \Omega} \varphi v \cdot n \mathrm{~d} s
$$

$\forall \varphi \in H_{1}$. By $(6.8)$ we can see that

$$
L^{*} v=0 \Longleftrightarrow \operatorname{div} v=0,\left.v \cdot n\right|_{\partial \Omega}=0 .
$$

Therefore, for the operators (6.7) and $(6.9)$ the vector fields $u$ in $H=$ $L^{2}\left(\Omega, \mathbb{R}^{n}\right)$ can be decomposed as

$$
u=\nabla \varphi+v, \operatorname{div} v=0,\left.v \cdot n\right|_{\partial \Omega}=0 .
$$

This is the Leray decomposition.

Example 6.2. The decomposition of the tensor in Riemannian manifolds. Let $M$ be a $n$-dimensional manifold without boundary, and $H=L^{2}\left(T_{r}^{k} M\right)$ be the space consisting of square integrable $(k, r)$-tensor on $M$, and

$$
H_{1}=H^{1}\left(T_{r}^{k-1} M\right) \quad\left(\text { or } H^{1}\left(T_{r-1}^{k} M\right)\right) .
$$

For the gradient operator

$$
L=\nabla: H_{1} \rightarrow H,
$$

the dual operator is the divergent operator

$$
L^{*}=\operatorname{div}: H \rightarrow H_{1}^{*} \text {. }
$$

By Theorem 6.1, for $u \in H=L^{2}\left(T_{r}^{k} M\right)$ we have

$$
u=\nabla \varphi+v, \operatorname{div} v=0, \varphi \in H_{1} .
$$

6.2. Variations with constrained-infinitesimals. Let $H, H_{1}$ be two Hilbert spaces, and

$$
L: H_{1} \rightarrow H, \quad L^{*}: H \rightarrow H_{1}^{*}
$$

be a pair of dual linear bounded operators. Consider the functionals defined on $H$, i.e.,

$$
F: H \rightarrow \mathbb{R}^{1} \text {. }
$$

Then we introduce the following definitions. Let

$$
\mathcal{N}^{*}=\left\{v \in H \mid L^{*} v=0\right\} \neq\{0\},
$$


i.e., $\operatorname{dim} \mathcal{N}^{*}>0$.

We now recall the notion of constraint variations given in Definition 2.1;

1) For any $u \in H$, the derivative operator of $F$ at $u$ with $L^{*}$-constraint, denoted by $\delta_{L^{*}} F(u)$, is defined as follows

$$
\left\langle\delta_{L^{*}} F(u), v\right\rangle_{H}=\left.\frac{\mathrm{d}}{\mathrm{d} t}\right|_{t=0} F(u+t v) \quad \forall v \in \mathcal{N}^{*} .
$$

2) The derivative operator of $F$ at $u$ with $L$-constraint, denoted by $\delta_{L} F(u)$, is defined as follows

$$
\left\langle\delta_{L} F(u), \varphi\right\rangle_{H_{1}}=\left.\frac{\mathrm{d}}{\mathrm{d} t}\right|_{t=0} F(u+t L \varphi) \quad \forall \varphi \in H_{1} .
$$

The following theorem is based on Theorem 6.1.

Theorem 6.2 (Variation with $L$-constraints). For the variational derivatives with $L^{*}$ and $L$ constraints defined in (6.15) and (6.16) we have the following conclusions.

1) For $\delta_{L^{*}} F(u)$, there is a $\varphi \in H_{1}$ such that

$$
\delta_{L^{*}} F(u)=\delta F(u)+L \varphi .
$$

2) For $\delta_{L} F(u)$, we have

$$
\delta_{L} F(u)=L^{*} \delta F(u) .
$$

Here $\delta F(u)$ is the normal derivative operator.

Proof. The theorem follows from Definition 2.1 and Theorem 6.1. In fact, by definition, we have

$$
\left\langle\delta_{L^{*}} F(u), v\right\rangle_{H}=\langle\delta F(u), v\rangle \quad \forall L^{*} v=0,
$$

which, by Theorem 6.1, implies 6.17.

Also, by definition, we have

$$
\left\langle\delta_{L} F(u), \varphi\right\rangle_{H_{1}}=\langle\delta F(u), L \varphi\rangle_{H}=\left\langle L^{*} \delta_{L} F(u), \varphi\right\rangle_{H_{1}},
$$

which leads to 6.18.

By Theorem 6.2, we shall show that all physical motion systems, without coupling to other systems, have a functional

$$
F: H \rightarrow \mathbb{R}^{1}
$$

such that the dynamical equations of the systems can be expressed as

$$
\frac{\mathrm{d} u}{\mathrm{~d} t}=-A \delta_{L} F(u)
$$


where $A$ is a coefficient matrix, $\delta_{L} F(u)$ is the derivative operator of $F$ at $u$ with $L$-constraint. Note that the normal derivative operator $\delta F(u)$ correspond to $\delta_{L} F(u)$ with $L=i d$ the identity.

\section{Dynamics of Other Physical Motion Systems}

7.1. Newtonian laws. A group of bodies with masses $\left(m_{1}, \cdots, m_{N}\right)$ motion in a potential field $V(x)$, the motion equations are

$$
\frac{\mathrm{d} p_{i}}{\mathrm{~d} t}=-m_{i} \delta_{i} V(x) \quad(1 \leq i \leq N)
$$

where $\delta_{i}=\partial / \partial x_{i}$. In (2.8), we have shown that (7.1) has the form of (2.6).

Consider the electron motion equation given by

$$
m \frac{\mathrm{d} v}{\mathrm{~d} t}=e E+\frac{e}{c} v \times H .
$$

Based on the electromagnetic theory,

$$
\begin{aligned}
& \text { momentum of electron } \quad p=m v+\frac{e}{c} \mathbf{A}, \\
& \text { electromagnetic potential } \quad \Phi=\varphi-\frac{1}{c} \mathbf{A} \cdot v \text {. }
\end{aligned}
$$

Then (7.2) is equivalent to the equation (see Landau and Lifshitz [1]):

$$
\frac{\mathrm{d} p}{\mathrm{~d} t}=-e \nabla \Phi
$$

which can be equivalently written as

$$
\frac{d u}{d t}=-J \delta \Psi
$$

where $u=(x, p)^{T}$, and

$$
\begin{aligned}
\Psi & =\frac{1}{2 m} p^{2}-\frac{e}{m c} \mathbf{A} \cdot p+\frac{e}{c} \mathbf{A}^{2}+e \varphi \\
J & =\left(\begin{array}{cc}
0 & -I \\
I & 0
\end{array}\right)
\end{aligned}
$$

7.2. Fluid Dynamics. We consider here two examples-the compressible Navier-Stokes equations and the Boussinesq equations. 
We start with the compressible Navier-Stokes equations:

$$
\begin{aligned}
& \frac{\partial u}{\partial t}+(u \cdot \nabla) u=\frac{1}{\rho}[\mu \Delta u-\nabla p+f], \\
& \frac{\partial \rho}{\partial t}=-\operatorname{div}(\rho u) \\
& \left.u\right|_{\partial \Omega}=0 .
\end{aligned}
$$

Let the constraint operator $L=-\nabla$ be the gradient operator, with dual operator $L^{*}=$ div. Also, let

$$
\Phi(u, \rho)=\int_{\Omega}\left[\frac{\mu}{2}|\nabla u|^{2}-f u+\frac{1}{2} \rho^{2} \operatorname{div} u\right] \mathrm{d} x .
$$

Then the equations of $(7.4)$ are written as

$$
\begin{aligned}
& \frac{\mathrm{d} u}{\mathrm{~d} t}=-\frac{1}{\rho} \frac{\delta_{L^{*}}}{\delta u} \Phi(u, \rho), \\
& \frac{d \rho}{d t}=-\frac{\delta}{\delta \rho} \Phi(u, \rho),
\end{aligned}
$$

which is in the form of 2.6 with coefficient matrix $A=\operatorname{diag}(1 / \rho, 1)$. Also, the pressure is given by

$$
p=p_{0}-\lambda \operatorname{div} u-\frac{1}{2} \rho^{2} .
$$

Here

$$
\frac{\mathrm{d} u}{\mathrm{~d} t}=\frac{\partial u}{\partial t}+\frac{\partial u}{\partial x_{i}} \frac{\mathrm{d} x_{i}}{\mathrm{~d} t}=\frac{\partial u}{\partial t}+(u \cdot \nabla) u, \quad \frac{d \rho}{d t}=\frac{\partial \rho}{\partial t}+u \cdot \nabla \rho .
$$

The Boussinesq equations are written as

$$
\begin{aligned}
& \frac{\partial u}{\partial t}+(u \cdot \nabla) u-\rho_{0}^{-1}[\nu \Delta u+\nabla p]=-g k \rho(T), \\
& \frac{\partial T}{\partial t}+(u \cdot \nabla) T-\kappa \Delta T=0, \\
& \operatorname{div} u=0,
\end{aligned}
$$

where $\nu, \kappa, g$ are constants, $u=\left(u_{1}, u_{2}, u_{3}\right)$ is the velocity field, $p$ is the pressure function, $T$ is the temperature function, $\bar{T}_{0}$ is a constant representing the lower surface temperature at $x_{3}=0$, and $k=(0,0,1)$ is the unit vector in the $x_{3}$-direction.

As before, we take the linear constraint operator $L=-\nabla$ with dual $L^{*}=$ div. Let

$$
\Phi(u, T)=\int_{\Omega}\left[\frac{\mu}{2}|\nabla u|^{2}+\frac{\kappa}{2}|\nabla T|^{2}\right] \mathrm{d} x .
$$


Then the Boussinesq equations of (7.6) are written as

$$
\begin{aligned}
\frac{\mathrm{d} u}{\mathrm{~d} t} & =-\frac{1}{\rho_{0}} \frac{\delta_{L^{*}}}{\delta u} \Phi(u, T)+B(u, T), \\
\frac{d T}{d t} & =-\frac{\delta}{\delta T} \Phi(u, T),
\end{aligned}
$$

which is in the form of 2.6 with coefficient matrix $A=\operatorname{diag}\left(1 / \rho_{0}, 1\right)$. The term $B(u, T)$ is given by

$$
B(u, T)=g \rho_{0} k \rho(T),
$$

which breaks the symmetry of the functional $\Phi(u, T)$, caused by the coupling between momentum equations and the temperature equation. Also, the incompressibility condition div $u=0$ is built into the construction of the basic function space, which we omit the details.

7.3. Hamiltonian dynamics. The principe of Hamiltonian dynamics (PHD) is a universal principle, which governs all energy conservative systems, including the astronomic mechanics, wave motion equations, quantum mechanics, and the Maxwell equations.

PHD amounts to saying that for a conservative system, there are two sets of conjugate functions $u=\left(u_{1}, \cdots, u_{N}\right), v=\left(v_{1}, \cdots, v_{N}\right)$ and a Hamiltonian energy $H=H(u, v)$, such that $u$ and $v$ satisfy the equations; see among others Ma and Wang [4]:

$$
\begin{aligned}
& \frac{\partial u}{\partial t}=k \frac{\delta}{\delta v} H(u, v), \\
& \frac{\partial v}{\partial t}=-k \frac{\delta}{\delta u} H(u, v),
\end{aligned}
$$

which can be expressed in form of 2.6 as

$$
\frac{\mathrm{d} v}{\mathrm{~d} t}=-A \delta H(u, v)
$$

where

$$
A=k\left(\begin{array}{cc}
0 & -I \\
I & 0
\end{array}\right)
$$

is an anti-symmetric matrix.

7.4. Lagrangian Dynamics. The principle of Lagrangian dynamics (PLD) is also a universal principle in physics, which governs the physical motion systems such as Newtonian mechanics, quantum physical system, elastic waves, classical electrodynamics etc; see among others [1, 4]. 
PLD amounts to saying that for an isolated motion system, there are state functions $u=\left(u_{1}, \cdots, u_{N}\right)$ and a functional $F(u)$ called the Lagrangian action, such that $u$ satisfies

$$
\delta F(u)=0,
$$

which can be equivalently referred to the Hamiltonian system (7.8) with conjugates $u$ and $v=u_{t}$.

\section{REFERENCES}

[1] L. D. Landau and E. M. Lifshitz, Course of theoretical physics, Vol. 2, Pergamon Press, Oxford, fourth ed., 1975. The classical theory of fields, Translated from the Russian by Morton Hamermesh.

[2] E. M. Lifschitz And L. P. Pitajewski, Lehrbuch der theoretischen Physik ("Landau-Lifschitz"). Band X, Akademie-Verlag, Berlin, second ed., 1990. Physikalische Kinetik. [Physical kinetics], Translated from the Russian by Gerd Röpke and Thomas Frauenheim, Translation edited and with a foreword by Paul Ziesche and Gerhard Diener.

[3] T. Ma and S. Wang, Phase Transition Dynamics, Springer-Verlag, 2013.

[4] —, Mathematical Principles of Theoretical Physics, Science Press, 2015.

[5] R. K. Pathria and P. D. Beale, Statistical Mechanics, Elsevier, third ed., 2011.

[6] L. E. ReichL, A modern course in statistical physics, A Wiley-Interscience Publication, John Wiley \& Sons Inc., New York, second ed., 1998.

(TM) Department of Mathematics, Sichuan University, Chengdu, P. R. CHINA

E-mail address: matian56@sina.com

(SW) Department of Mathematics, Indiana University, Bloomington, IN 47405

E-mail address: showang@indiana.edu 